\title{
WEST NILE VIRUS SYSTEM DYNAMICS INVESTIGATION IN DALLAS COUNTY, TX
}

\author{
Mohammad F. Obeid \\ John Shull \\ Modeling, Simulation, and Visualization Engineering Department \\ Old Dominion University \\ Norfolk, VA 23508 USA
}

\begin{abstract}
After its first introduction in 1999, West Nile Virus (WNV) has spread very widely along the east coasts of the United States before appearing in Texas where 1792 cases were reported of which 82 were fatal in 2012. The interesting patterns and behavior of the virus and its amplified impact on the county of Dallas drove this work. This paper encompasses a thorough development of a systems dynamics simulation model that imitates the virus's infectious behavior and dynamics in Dallas County, TX utilizing historical data collected and the aid of suitable software packages.
\end{abstract}

\section{INTRODUCTION}

System dynamics is an approach aimed to understand the behavior of complex systems over time and is a very suitable tool to model diseases. Systems are represented using feedback loops and time delays as well as stocks and flows that graphically describe the model (S.C. Brailsford 2007, Erik R. Larsen 1997). The method is based on understanding the structure of any system which might be composed of circular, interrelated, interlocking and sometimes time-delayed relationships. In order to represent system dynamics, a causal loop diagram is constructed first to illustrate all the system components and interactions all connected with positive and negative reinforcement feedback loops which indicate a quantity increase or decrease respectively. After that, a stock-flow diagram is constructed to visualize and analyze the system's behavior in a quantitative manner. A stock is the population or quantity that increases or decreases over time whereas the flow is the rate of change in a stock (Denis R. Towill 1993, Kirkwood 2013, Fishwick 1995).

West Nile Virus is a mosquito-borne disease that was first identified in the West Nile sub region in Uganda in 1937. CDC reported 30,491 cases of WNV as of 2010 of which 1196 were reported fatal. Most experts indicate that these numbers understate the reality since the symptoms of this disease are very similar to other viral infections and therefore might be falsely diagnosed or pass unnoticed as approximately $80 \%$ of WNV infection cases in humans are subclinical which cause no symptoms.

It is well established that dry weather conditions coupled with heat waves amplify the activity and life cycle of the disease and increase the infection probability since it drives birds, which is considered the primary host of WNV, to get closer to shrinking water sites where the virus is usually more active. Furthermore, drought also reduces the activity of mosquito predators such as frogs and dragonflies which increases the life span of the virus (AMCA 2011, Epstein 2001, Janousek and Olson 2006, McLean 2006).

The virus's first detection in New York in 1999 followed by its sudden appearance in Florida in 2001 and in Texas in 2002 has motivated a lot of studies. The patterns observed of the virus spread over several distant locations was best explained to be directly related with birds' migration as they serve as long distance travelling agents. Many studies have indicated that the virus's activity is triggered when temperature exceeds a threshold of around 13 degrees Celsius, below which a mosquito can carry the virus but not 


\section{Obeid and Shull}

show symptoms and not even be affected by it and it could even survive a whole winter if the temperature stayed as low as suitable (overwintering). When a mosquito gets infected after a blood meal, it doesn't become infectious and doesn't lay infected eggs until an incubating period passes. Such a period also exists in humans and birds and it is only when this period ends that the virus can get transmitted. Only a female mosquito with WNV titer greater than 3.5Log10 PFU is capable of disease transmission. (McLean 2006, Pollock 2008, Rappole, Derrickson and Hubalek 2000, West Nile Virus Human Disease Map 2012, Reisen, Fang, and Martinez 2006).

In this work, a conceptual model of the virus's activity in Dallas County is constructed and required data is collected to build a simulation model representing the system's behavior which will be used to analyze its properties and its future trends.

\section{METHODOLOGY}

Generally speaking, there are two overlapping stages when it comes to system dynamics modeling. The first stage is the conceptual model where the system is defined and conceptualized and the second one is the analytical model where the model's behavior and properties are analyzed in addition to an evaluation against provided data (Towill 1993).

\subsection{Conceptual Model (Causal-Loop)}

The system can be broken into three major sectors or areas each containing multiple loops. They are human cycle, bird cycle and lastly the mosquito cycle which contains a sub cycle of eggs' life cycle. Figure 1 illustrates the conceptual model representing the system in a Causal-Loop diagram.

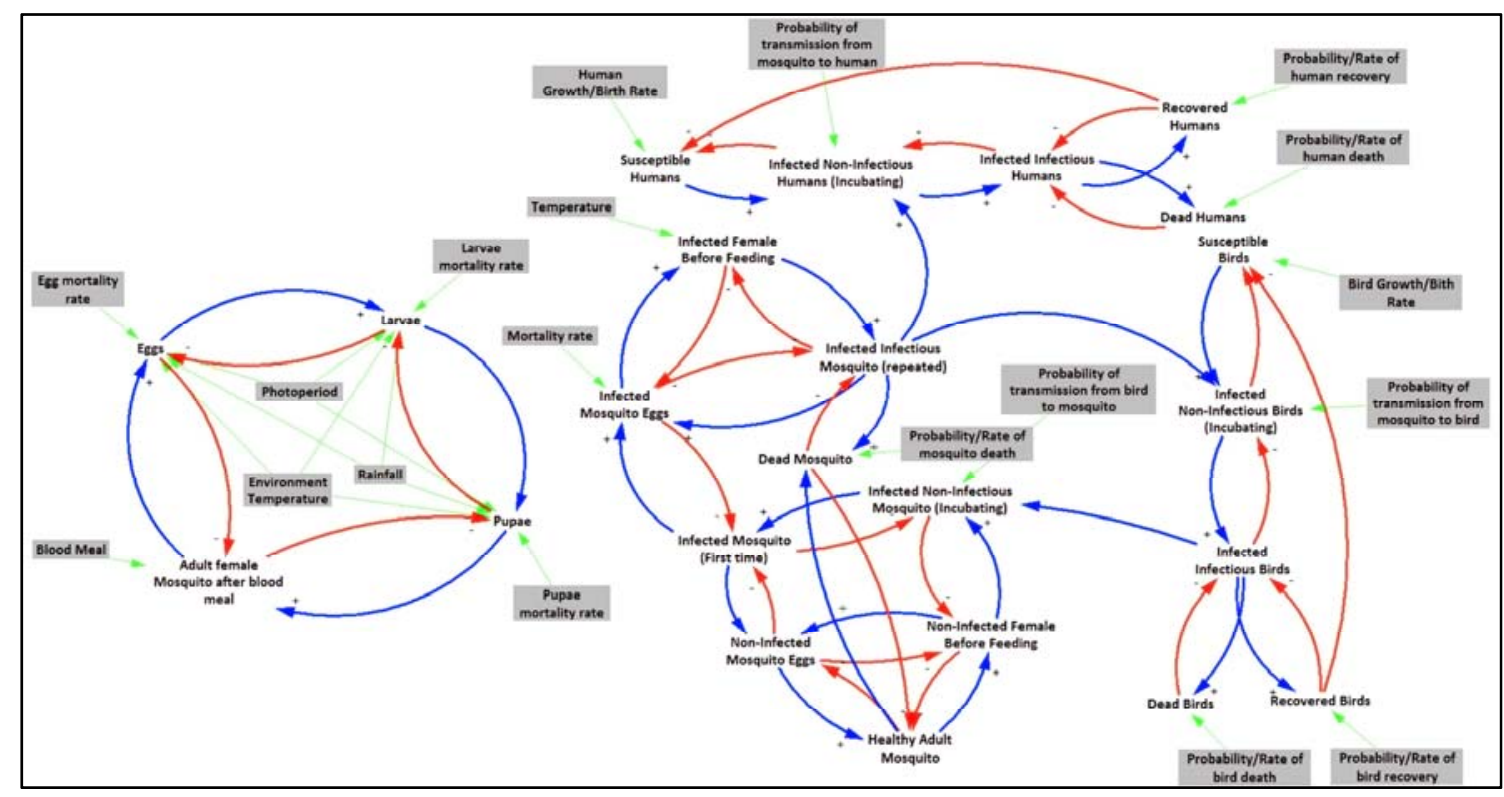

Figure 1: Causal-Loop diagram of the model showing all involved cycles and variables

\subsubsection{Human Cycle}

The cycle starts with a population of susceptible humans which is affected by the human growth rate (birth - death); from this stage a transition to the infected incubating human population is affected by the human infection rate. After the incubation period, a transition is made to the infected infectious human population from which a transition occurs to either a recovery or a death state according to recovery and death rates. Each transition that occurs increases the level of the next population and, in turn, decreases 


\section{Obeid and Shull}

the level of the previous population which is demonstrated in the causal loop diagram by a positive blue arc moving forward and a negative red arc moving backward respectively and this is the case for all the loops for all of the cycles.

\subsubsection{Bird Cycle}

The cycle starts with susceptible birds population that grows according to the bird growth rate, a transition is made to the infected incubating bird population in accordance with the bird infection rate. After the incubation period takes place a transition is made either to the infected infectious bird population from which a recovery or death can occur according to bird recovery and death rates. The infected infectious population of birds is related/connected to the infected mosquito population (in the mosquito cycle) which demonstrates the mosquito's feeding process (blood meal).

\subsubsection{Mosquito Cycle}

The cycle starts with the healthy mosquito population before feeding. This population feeds the mosquito healthy eggs population which feeds back into the healthy adult mosquito population. The healthy mosquito population also transitions to an infected incubating mosquito population affected by the virus transmission rate from bird to mosquito. According to (S.C. Brailsford 2007), an infected mosquito can lay both infected and non-infected eggs depending on the incubation period compared to the deposition period. But typically, after the first batch of eggs, an infected mosquito lays only infected eggs.

\subsubsection{Mosquito Sub-cycle}

The cycle starts with an adult female after feeding on a host and laying eggs. An egg transitions to each succeeding stage of larvae, pupae and then adult mosquito if temperature, duration of light exposure and amount of rainfall were all suitable.

\subsection{Data Collection and Model Construction (Stock-Flow)}

After building a conceptual model, data about Dallas County was collected from several sources regarding initial populations, weather conditions, rates and many others as well as equations and functions that were constructed mathematically to have the model behave as close to reality as possible. Before describing the data, used terminology in the model is now defined.

Parameters are values that are constructed into the model before it is run. A Stock is a variable measured at a specific instance and represents a measure or quantity at that point in time and have accumulated from previous time steps. An Auxiliary is a variable that has an equation built into it and converts any value that passes through it accordingly. A Flow is a variable representing a rate or a speed between two stocks. A Delay is an amount of time that affects the simulation clock. A Function is an equation that stores information and can be retrieved whenever that function is called (Denis R. Towill 1993, Kirkwood 2013, Fishwick 1995).

\subsubsection{Functions Associated with System Dynamics Model}

All functions will be described first within this section separately:

- WeatherData2011(): contains tabular data regarding temperature (in degrees Celsius) for 365 days collected from 2011 weather data in Dallas County.

- Daylight2011(): contains tabular data regarding the amount of daylight hours each day for 365 days collected from 2011 weather data for Dallas County.

- Rain2011(): contains tabular data regarding daily rainfall for 365 days collected from 2011 weather data for Dallas County. 


\section{Obeid and Shull}

- DailyBirdMigIn(): contains data that was taken from the 2011 www.ebird.org data set which is a report of eye witness accounts for species of birds that were spotted migrating into Dallas County (Ebird.org 2012).

- DailyBirdMigOut(): contains similar data to the previous function only regarding birds migrating out of Dallas County.

- DailyFeedingProbability(): changes the value of the mosquito's probability of feeding according to the current temperature of the day.

- HourlyFeedingWindow(): On any given day, mosquitoes are more prone to feed when the sun is not out, the dark. This function pulls tabular data that stores hours of sun exposure, subtracts this pulled data from 24 hours then divides the result by 24 hours to represent the information as a percentage of the day that is considered a "feeding window".

- PovertyFunction(): calculates the percentage of Dallas County's residents who are considered under poverty line (according to poverty rate of $17.6 \%$ ), residents who are continuously exposed to mosquitoes because of their work nature or those who fall into the age category considered most venerable to WNV (over 65 of age or are very young) (U.S. Department of Commerce 2011).

- LarvaetoAdultMosq(): returns the probability of associated rates of larvae to full adult given the current time.

After explaining the functions that are be used across all the cycles, an explanation of what composes each cycle is provided. Each cycle's data will be described separately and broken down into parameters, stocks, flows and auxiliaries.

\subsubsection{The Human Cycle Breakdown}

\subsubsection{Human Cycle Parameters}

- Initial Human Population $\left(\right.$ Pop $\left._{H H}\right)=2011$ Dallas TX Population $=2,416,014$ (U.S. Department of Commerce 2011).

- Dallas Daily Growth Rate $\left(D G R_{\text {Dallas }}\right)=\frac{\text { Current Popul. }- \text { Last Year Popul. }}{365}=139$.

- Human Infection Probability $\left(P_{H I}\right)=0.01$. As even bitten by a highly contagious mosquito a person will still have a ball park average probability of getting the disease that is $1 \%$ (Texas Department of State Health Services 2013).

\subsubsection{Human Cycle Stocks}

Susceptible Humans Population $\left(\mathrm{Pop}_{H H}\right)=2,416,014$. The rest of the stocks in this cycle are Infected Humans Population $\left(P o p_{H I}\right)$, Dead Humans Population $\left(P o p_{H D}\right)$ and Recovered Humans Population $\left(\mathrm{Pop}_{H R}\right)$ and are all initiated at zero for the simulation.

\subsubsection{Human Cycle Auxiliaries}

- Probability that the host is Human $\left(P_{\text {HostH }}\right)$ is Triangular $(0.05,0.40,0.33)$. As (Vinogradova 2000) states, in a similar study of similar temperature and weather conditions the probability that a mosquito will feed on a mammal is between $33 \%$ and $40 \%$. Since this value includes all mammals, variability was established using the triangular distribution to compensate for that difference stochastically.

- Probability of Mosquito biting Human $\left(P_{B i t e H}\right)$ is shown in (1):

$P_{\text {BiteH }}=\left(\right.$ DailyFeedingProbability (time) $* P_{H o s t H} * P_{H I} *($ HourlyFeedingWindow (time $\left.\left.)\right)\right) *$

$\frac{\text { InfectedMosquito }}{\text { InfectedMosquito+HealthyMosquito }}$. 


\section{Obeid and Shull}

This was constructed as a four-part conditional probability multiplied by the total infected mosquito population and is explained as follows:

1. Probability of feeding, given the current temperature (the function DailyFeedingProbability()is called and is fed the value of the current time). Generally in warmer conditions the mosquitos attack at a higher rate.

2. Probability that the host is human.

3. Human Infection Probability.

4. Time window of which a mosquito generally feeds (morning and night, just before sunrise and sunset) by calling the function HourlyFeedingWindow() which has tabular data that is computed to output a percentage of the day that is associated with no light.

Associated with this flow is a Delay in time, meaning that this rate/probability will only take effect after this delay. So the number of humans passing through the flow that implements this auxiliary will not reach the next stock until this time passes. This is based upon the NYC health department Delay Period of 3 to 15 days (NYC Health 2010).

\subsubsection{Human Cycle Flows}

- Human Infection Rate $\left(R I_{H}\right)$ (Susciptible Humans to Infected Human) is shown in (2):

$$
R I_{H}=\text { PovertyFunction }\left(\left(D G R_{\text {Dallas }}+\text { Pop }_{H H}\right)\right) * P_{H I} .
$$

Variables involved are:

1. Function: PovertyFunction().

2. Parameter: Dallas Daily Growth Rate.

3. Stock: Susceptible Humans.

4. Auxiliary: probability of Mosquito biting Human.

- Human Death Rate $\left(R D_{H}\right)$ (Infected Humans to Dead Humans) $=0.0493 *$ Pop $_{H I}$.

Variables involved are:

1. Stock: Infected Humans.

2. A constant $=0.0493$ which is associated with the probability of Dallas County's current mortality rate of those that have advanced into a serious state of the West Nile Virus (Texas Department of State Health Services 2013).

- Human Recovery Rate $\left(R R_{H}\right)$ (Infected Human to Recovered Humans) $=0.9507 * P_{o p} p_{H I}$.

Variables involved are:

1. Stock: Infected Humans.

2. A constant $=1-0.0493$, which is the associated probability of not having the West Nile Virus advanced into a serious condition.

\subsubsection{The Bird Cycle Breakdown}

\subsubsection{Bird Cycle Parameters}

- Initial Bird Population (InitialPop ${ }_{B}$ ) for Dallas (Rocky Mountain Bird Observatory 2007) is shown in (3):

$$
\text { InitialPop } p_{B}=\frac{\text { Total Bird Population of Texas }}{\text { Total Square Miles }} * \text { Dallas Area }=\frac{188,778,410}{261,231.71} * 871.28=629628.20 \text {. }
$$

- Probability that the Host is a Bird $P_{H o s t H}=0.60$. According to (Vinogradova 2000), a study was done on a population of mosquitoes that are identical to the mosquitoes of Texas and have identical conditions, this study showed that in dense and warm areas, mosquitoes tend to feed more on 


\section{Obeid and Shull}

humans and less on birds than when in a rural setting, in which this case the mosquitoes feed almost entirely on the birds for their blood meal.

\subsubsection{Bird Cycle Stocks}

- Susceptible Bird Population ( $\left(\mathrm{op}_{B H}\right.$ ) (Rocky Mountain Bird Observatory 2007) is shown in (4):

$$
P_{\text {op }}{ }_{B H}=629628.206+\text { Healthy Migrating(in) Birds - Migrating(out) Birds. }
$$

The rest of the stocks in this cycle are Infected Birds Population ( Pop $\left._{B I}\right)$, Dead Birds Population $\left(P o p_{B D}\right)$ and Recovered Birds Population $\left(P o p_{B R}\right)$ and are all initiated at zero for the simulation.

\subsubsection{Bird Cycle Auxiliaries}

- Probability of Bird Migrating with WNV $\left(P_{M i g w W N V}\right)=$ Triangular $(0.01,0.35,0.10)$. This is the probability associated with any migratory bird passing through Dallas TX carrying WNV.

- $\quad$ Migrating Sick Bird = DailyBirdMigIn $(\operatorname{time}()) * P_{M i g w W N V}$.

This was constructed by calling the function DailyBirdMigIn() that uses tabular data of the year to allocate a quantity of birds that have come into Texas on this given day, this value is then multiplied by the probability of already being a carrier of WNV.

- $\quad$ Migrating Healthy bird = DailyBirdMigIn $(\operatorname{time}()) *\left(1-P_{\text {MigwWNV }}\right)$.

This was constructed by calling the function "DailyBirdMigIn()" again and multiplying the value by the probability of not being a carrier of WNV.

\subsubsection{Bird Cycle Flows}

- Bird Infection Flow (between Healthy Birds and Infected Birds) is given in (5):

$$
\text { Bird Infection Flow }=\left(R_{\text {Trans }} * P_{\text {Oop }}\right)+\text { Migrating Sick Bird. }
$$

Variables involved are:

1. Auxiliary: Migrating Sick Birds.

2. Auxiliary: Transmission Rate $\left(R_{\text {Trans }}\right)$ which will be constructed in the mosquito cycle.

3. Stock: Healthy Bird Population

- Bird Death Rate $\left(R D_{B}\right)$ (between infected birds and dead birds) $=$ Pop $_{B I} *$ BirdDeathProb. Variables involved are:

1. Auxiliary: Bird Death Probability is a stochastic Triangular $(0.05,0.95,0.69)$.

2. Stock: Infected Bird Population.

- Bird Recovery Rate $\left(R D_{B}\right)=(1-$ BirdProbabilityofDeath $) *$ InfectedBird.

The variables involved are the same as the Bird Death rate, but using the death probability's complementary to 1 as the bird's recovery probability.

\subsubsection{The Adult Mosquito Cycle Breakdown}

\subsubsection{Adult Mosquito Cycle Parameters}

- Daily Feeding Habit $\left(P_{F e e d}\right)=0.80$. According to (Vinogradova 2000), Mosquitoes have an average daily probability of feeding on any given host $0.80 \%$.

- Initial Mosquito Population $\left(\right.$ InitialPop $\left._{M}\right)=10,000$. This number was used in a previous study.

\subsubsection{Adult Mosquito Cycle Stocks}

- Susceptible Mosquito Population $\left(\operatorname{Pop}_{M H}\right)=10,000$. Its initial value changes according to (6): 
Obeid and Shull

$\frac{d\left(P_{0} p_{M H}\right)}{d t}=$ YoungAdults - HealthyMosquitoesDeathRate - MosquitoInfectionFlow.

Variables involved are:

1. Young Adults: new Healthy Mosquito Eggs that have hatched.

2. Mosquito Healthy Death Rate: the natural life span of the mosquito.

3. Mosquito Infection Flow: mosquitoes that contract WNV.

- Infected Mosquito Population $\left(P_{0 p_{M I}}\right)=P_{o p_{M H}} *$ TransovarialTransmissionRate. Its initial value changes according to (7):

$\frac{d\left(\text { Pop }_{M I}\right)}{d t}=$ MosquitoInfectionFlow + YoungInfectedAdults - MosquitoDeathRate .

In addition to the Mosquito Infection Flow and the Mosquito death Rate, the variable Young Infected Adults is involved here which is the new mosquitoes that just hatched from an infected egg. Dead Mosquito Population is initiated as zero for the simulation.

\subsubsection{Adult Mosquito Cycle Auxiliaries}

- Transovarial Transmission Rate $=0.01$. According to (Rappole, Derrickson and Hubalek 2000), 0.01 is the percentage of mosquitoes that survived through the overwintering period.

- $\quad$ Transmission Rate $=$ DailyFeedingProbability $($ time $) * P_{H o s t B} *\left(\frac{{ }^{P o p_{M I}}}{\text { Pop }_{M I}+P_{\text {op }}}\right)$.

- This was constructed using a set of probabilities:

1. The function DailyFeedingProbability() is called to represent the probability of daily feeding given the current time.

2. Probability that the host is a bird $=0.60$.

3. The proportion of infected mosquitoes to the entire population of mosquitoes.

- Mosquito Infection Rate $\left(R I_{B}\right)$ is given in (8):

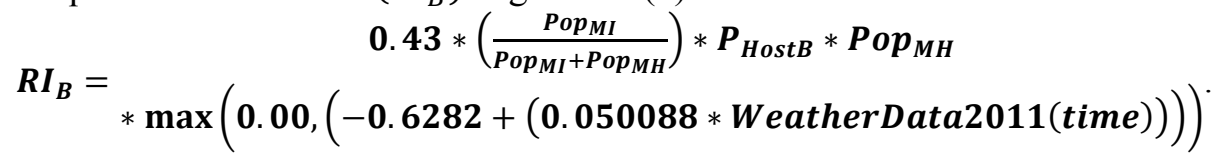

- This was constructed using a set of probabilities:

1. Constant $=0.43$, which is the transmission rate from an infected bird to a mosquito.

2. Probability that the host is a bird.

3. Temperature probability of WNV being sustainable by calling the "WeatherData2011()" function.

4. The proportion of infected birds to total bird population.

This would result in a rate of infection for mosquitoes in accordance to what day it is on the simulation clock. When this value is used in a the Flow, it will not take effect immediately as a Delay was programmed and is vindicated upon the temperature. This delay represents the period of time for which the mosquito is infected-but cannot pass on the virus.

- Mosquito Death Probability $\left(P_{\text {DeathM }}\right)=0.13$ which is the probabilty of a natural death for the mosquito (Paterson 2012).

\subsubsection{Adult Mosquito Cycle Flows}

- Mosquito Infection Flow (between Healthy Mosquito and Infected Mosquito). The variable involved is the Mosquito Infection Rate auxiliary.

- Mosquito Death Rate (between Infected Mosquito and Dead Mosquito) $=P_{\text {DeathM }} *$ Pop $_{M I}$. 
Variables involved are:

1. Auxiliary: Mosquito Death Probability.

2. Stock: Infected Mosquito.

- Healthy Mosquito Death Rate (Healthy Mosquito to Dead Mosquito) $=P_{\text {DeathM }} *$ Pop $_{M H}$. As healthy mosquitos have a probability of natural death as well.

\subsubsection{Mosquito Egg Cycle Breakdown}

\subsubsection{Mosquito Egg Cycle Stocks}

- Eggs from Healthy Mosquito: initially set to zero.

- Eggs from Infected Mosquito: initially set to zero.

- Born Healthy Adult $\left(\right.$ Adult $\left._{H}\right)$ : initially set to zero. Its initial value changes according to (9):

$$
\frac{d\left(\text { Adult }_{H}\right)}{d t}=\text { EmergenceRateHealthy }- \text { YoungHealthyAdults. }
$$

Variables involved here are:

1. EmergenceRateHealthy $\left(R_{e m H}\right)$ : number of healthy eggs that made it through the process.

2. YoungHealthyAdults: quantity of hatched adults from this population of eggs.

- Born Infected Adult $\left(\right.$ Adult $\left._{I}\right)$ : initially set to zero. Its initial value changes according to (10):

$$
\frac{d\left(\text { Adult } M_{I}\right)}{d t}=\text { EmergenceRateInfected }- \text { YoungInfectedAdults } \text {. }
$$

Variables involved here are:

1. EmergenceRateInfected $\left(R_{\text {emI }}\right)$ : number of infected eggs that made it through the process.

2. YoungInfectedAdults: quantity of hatched Adults from this population of eggs.

\subsubsection{Mosquito Egg Cycle Auxiliaries}

Only one Auxiliary is involved here. Egg to Larvae Emergent Rate $\left(R_{E t o L}\right)=0.62447$. According to (Kristen Bartlett-Healy 2012), a sample of 25 rural homes yielded 80 containers (3.2 containers per house unit) had a $56 \%$ probability of containing larvae whereas another sample of 25 urban homes yielded 116 containers (4.64 containers per house unit) had a $68.2 \%$ probability of containing larvae. Dallas County has 94402 home units of which 532562 are considered rural which indicates that there are $532562 * 3.2=$ 1704198.4 containers of which $1704198.4 * 0.56=954351.104$ are viable to support larvae life. On the other hand, there are 411593.592 home units considered urban which indicates that there are $411593.592 * 4.64=1909794.26$ containers of which $1909794.26 * 0.682=1302479.69$ are viable to support larvae life. This totals in 2256830 containers of which 3613992.66 viable to support life for larvae giving a 0.62447 probability that the eggs laid will turn into larvae.

\subsubsection{Mosquito Egg Cycle Flows}

- Emergence Rate Healthy $\left(R_{e m H}\right)$ (from Healthy Eggs to Healthy Adult) is given in (11):

$\boldsymbol{R}_{\text {EtoL }} *$ EggsFromHealthyMosquito $*$ LarvaetoAdultMosq(time $)$.

Variables involved here are:

1. Auxiliary: Egg to Larvae Emergence Rate.

2. Stock: Eggs from healthy mosquito.

3. Function: LarvaetoAdultMosq(). 


\section{Obeid and Shull}

This equation also uses a Delay to represent the associated incubation period from egg to adulthood.

- Emergence Rate Infected ( $R_{\text {emI }}$ ) (from Infected Eggs to Infected Adult) is given in (12):

$$
\boldsymbol{R}_{\text {EtoL }} * \text { EggsFromInfectedMosquito } * \text { LarvaetoAdultMosq(time). }
$$

Variables involved here are:

1. Auxiliary: Egg to Larvae Emergent Rate.

2. Stock: eggs from infected mosquito.

3. Function: LarvaetoAdultMosq().

This equation uses a Delay identical to the Emergence Rate Healthy.

- Young Healthy Adults (from Born Healthy Adult to Healthy Mosquito).

- Young Infected Adults (from Born Infected Adult to Infected Mosquito). The value involved here is the quantity that is considered born and is infected which is added to the current infected mosquito population.

\section{SIMULATION AND RESULTS}

AnyLogic software was used to build the model as described in the methodology. Using built-in functionalities for system dynamics models as well as the capability to construct functions and if-statements, all the parameters, stocks, flows, auxiliaries and functions were developed in a manner that mimics the real system and all entities were interconnected with relationships where needed (Figure 2).

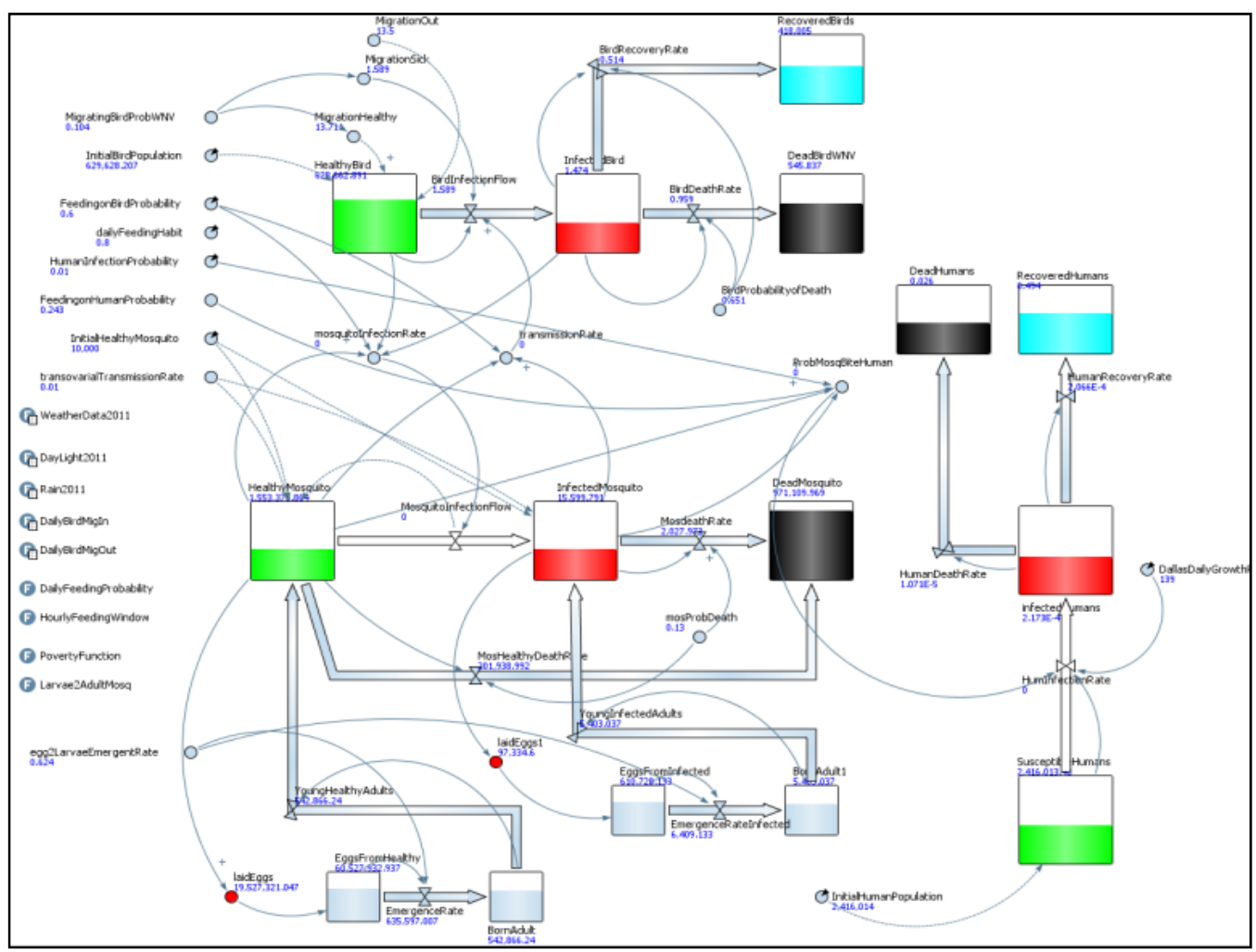

Figure 2: A snapshot from the constructed Simulation Model using AnyLogic 


\section{Obeid and Shull}

The simulation was run for 365 days to illustrate the system's behavior in a whole year and accumulated results were obtained. The system showed a very natural pattern as the mosquito eggs were increasing the adult population which affected the infected populations and thus infecting birds and humans using closed feedback loops. Figures 3, 4 and 5 show some results regarding and the change in number of infected humans over time, the change in number of infected birds over time and the accumulated number of human deaths at the end of the 365 days respectively.

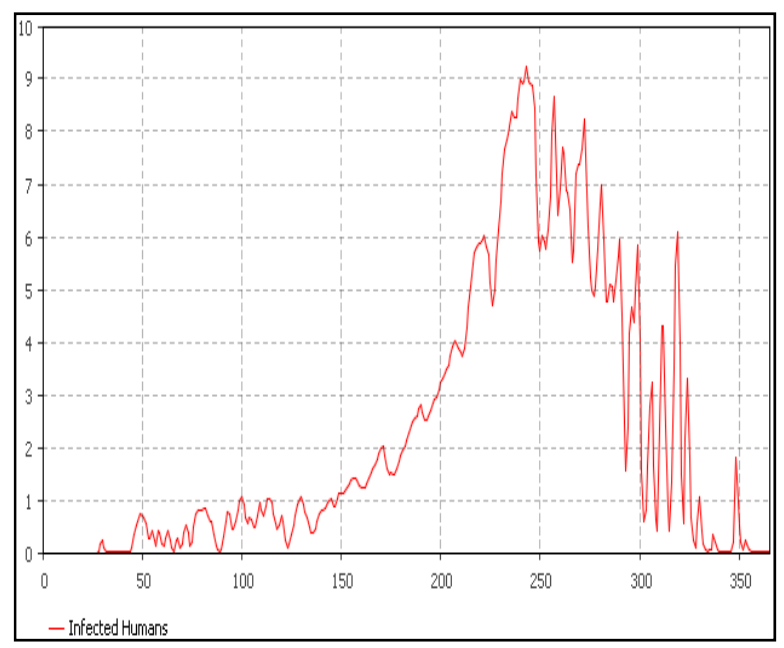

Figure 3: Simulation results of number of infected humans over time

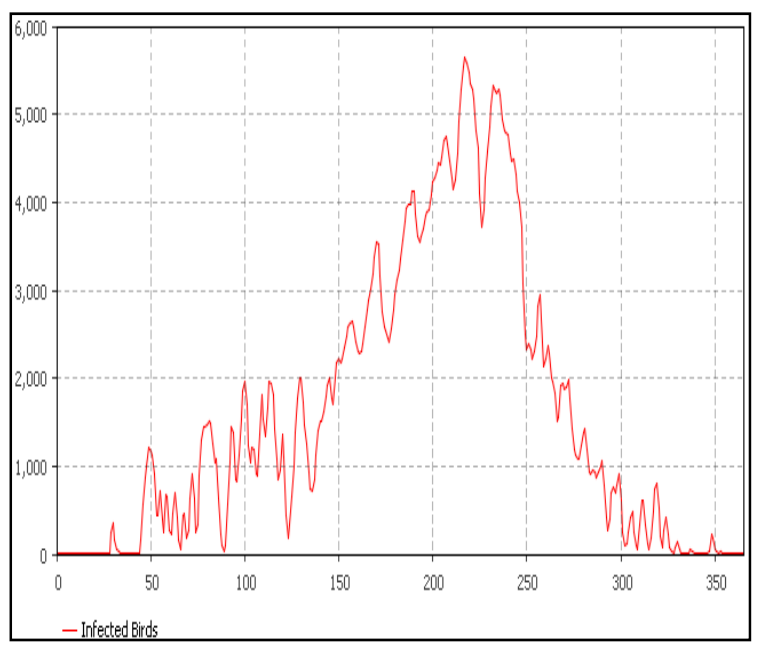

Figure 4: Simulation results of number of infected birds over time

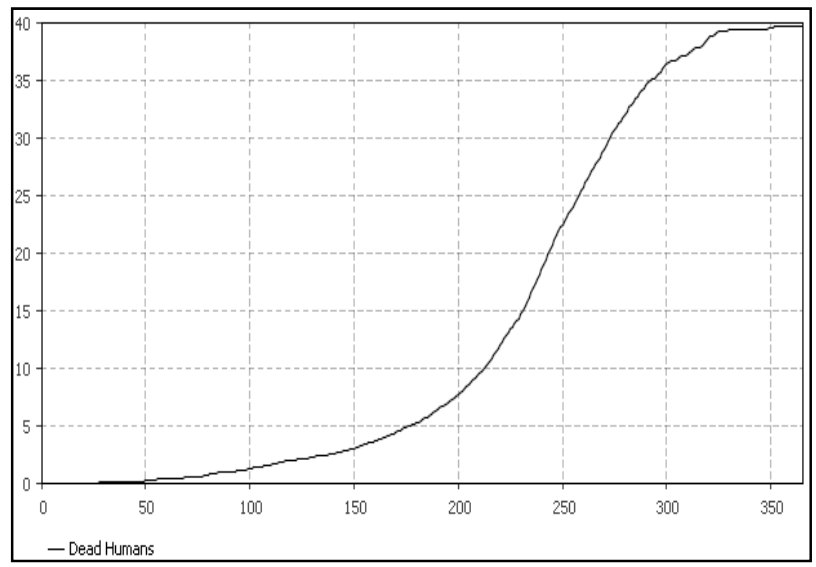

Figure 5: Simulation results of number of human deaths after a one year run.

\section{VALIDATION}

Many studies have used data collection using bird traps and mosquito sampling as well as analyzing health reports to show actual results of the virus's behavior. It is well established that the virus's activity peaks between the months of June and September (Epstein 2001, Texas Department of State Health Services: West Nile Virus in Texas 2013, Dennett JA 2007, S.C. Brailsford 2007). This work has produced results that show a very similar trend and spread of data which would validate that the model is a 


\section{Obeid and Shull}

true representation of the real system's nature. This can be observed by comparing Figures 3 and 4 with Figures 6 and 7. In addition, the simulation resulted in 39 human deaths while in real life, around 35 fatal WNV cases were reported in Dallas county which also helps validate the model's logic and variables used.

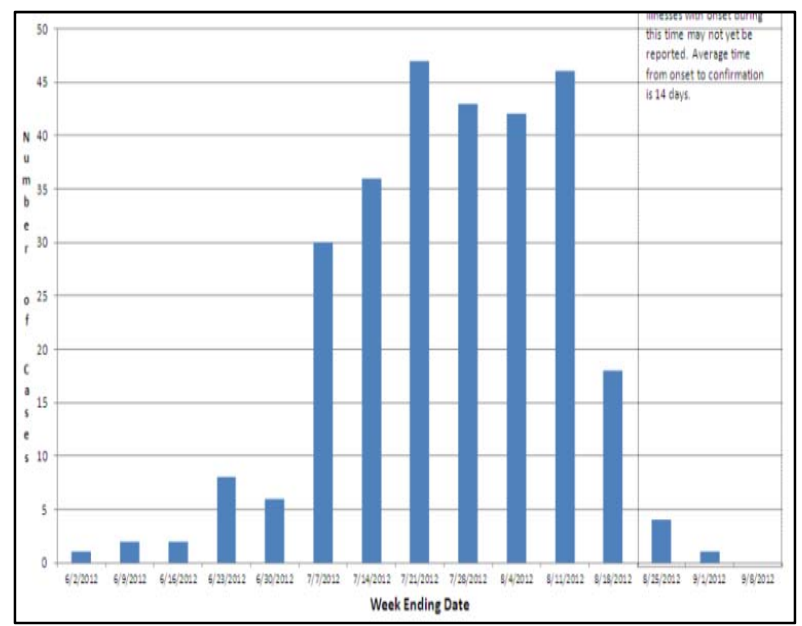

Figure 6: Number of confirmed WNV cases by week of Onset in Dallas County, 2012 (Texas Department of State Health Services 2013)

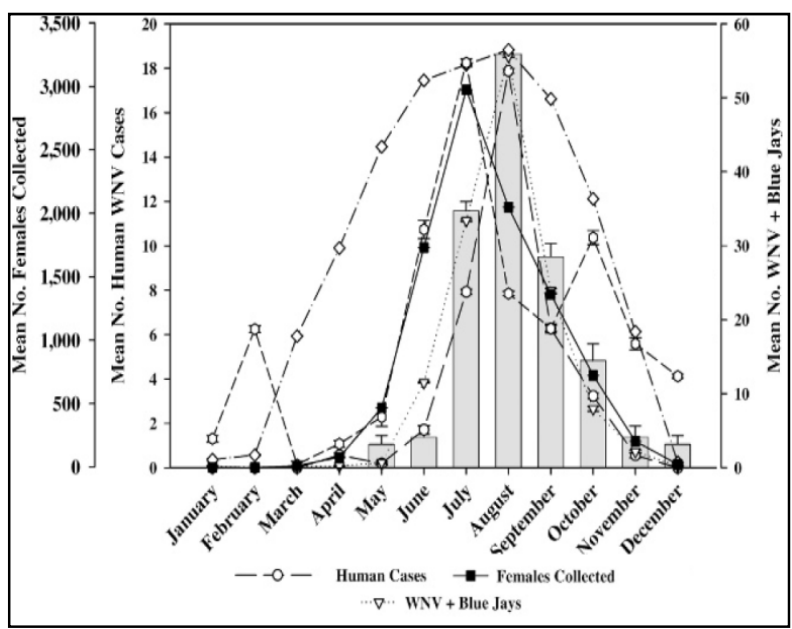

Figure 7: An overlay of female Aedes albopictus mosquito collected, reported WNV cases of humans and Blue Jays in 2006 (Dennett JA 2007)

\section{CONCLUSION}

A system dynamics simulation model was built to illustrate the behavior and impact of the West Nile Virus on Dallas County, TX. Variables and parameters were constructed utilizing data collected from several sources and mathematical functions were developed to mimic the mosquito's life cycle, the virus infectious behavior as well as the impact on the bird and human susceptible populations. Obtained results were comparable with actual results and conclude the validity of the model and motivate future and further development to be used for sensitivity analysis as well as forecasting.

\section{REFERENCES}

AMCA. Mosquito-Borne Diseases. 2011. http://www.mosquito.org/mosquito-borne-diseases (accessed November 22, 2012).

Dennett JA, Bala A, Wuithiranyagool T, Randle Y, Sargent CB, Guzman H, Siirin M, Hassan HK, Reyna-Nava M, Unnasch TR, Tesh RB, Parsons RE, Bueno R Jr. "Associations between two mosquito populations and West Nile virus in Harris County, Texas, 2003-06." Journal of the American Mosquito Control Association 23, no. 3 (2007): 264-275.

Ebird.org. EBird. 2012. www.ebird.org (accessed 2012).

Epstein, Paul. "West Nile Virus and the Climate." Journal of Urban Health, 2001: 367-371.

Erik R. Larsen, Ann van Ackere, Kim Warren. "The growth of service and the service of growth: Using system dynamics to understand service quality and capital allocation." Decision Support Systems, 1997: 271-287.

Fishwick, Paul A. Simulation model design and execution : building digital worlds. Englewood Cliffs, N.J.: Prentice Hall, 1995.

Janousek, Thomas, and J Olson. "Seasonal Variations in Activity and Size of Adult Females and Local distribution of Larvae for Populations of Culex Salinarius in the Upper Coastal Zone of Southeastern Texas." BioOne, 2006: 47-53. 


\section{Obeid and Shull}

Kirkwood, Craig W. System Dynamics Methods: A Quick Introduction. Arizona State University, Jan 12, 2013.

Kristen Bartlett-Healy, Isik Unlu, Peter Obenauer, Tony Hughes, Sean. "Larval Mosquito Habitat Utilization and Community Dynamics of Aedes albopictus and Aedes japonicus." Journal of Medical Entomology 49 (2012): 813-824.

McLean, Robert. "West Nile Virus in North American Birds." Ornithological Monographs, 2006: 44-64.

Mecoli, M., V. Angelis, R. Berchi, and S. Brailsford. System Dynamics models to assess the risk of mosquito borne diseases and to evaluate control polices. Rome: University of La Sapienza, 2007.

NYC Health. West Nile Virus FAQ. 2010. http://www.nyc.gov/html/doh/html/wnv/wnvfaq1.shtml.

Paterson, A. N. Clements and G. D. "The Analysis of Mortality and Survival Rates in Wild Populations of Mosquitoes." Journal ofApplied Ecology 18 (2012): pp. 373-399.

Pollock, Christal. "West Nile Virus in The Americas." BioOne, 2008: 151-157.

Rappole, John, Scott Derrickson, and Zdenek Hubalek. "Migratory Birds and Spread of West Nile Virus in the Western Hemisphere." Emerging Infectious Diseases, 2000: ISSN: 1080-6059.

Reisen, William, Ying Fang, and Vincent Martinez. "Effects of Temperature on the Transmission of West Nile Virus by Culex tarsalis." BioOne, 2006: 309-317.

Rocky Mountain Bird Observatory. PIF landbird Population Estimates Database. January 2007. http://rmbo.org/pif_db/laped/ (accessed November 21, 2012).

S.C. Brailsford, R. Berchi, V. De Angelis, M. Mecoli. System Dynamics models to assess the risk of mosquito borne diseases and to evaluate control polices. Rome: University of La Sapienza, 2007.

Texas Department of State Health Services. West Nile Virus in Texas. Sept 17, 2013. http://www.dshs.state.tx.us/idcu/disease/arboviral/westnile/.

Towill, Denis R. "System dynamics- background, methodology, and applications." Computing \& Control Engineering Journal 4 (1993): 201- 208.

U.S. Department of Commerce. State \& County Quick Facts: Dallas County, Texas. Washington DC: United States Census, 2011.

Vinogradova, Elena. Culex Pipiens Pipiens Mosquitoes: Taxonomy, Distribution, Ecology, Physiology, Genetic, Applied Importance and Control. Pensoft Publishers, 2000.

West Nile Virus Human Disease Map. November 20, 2012. http://diseasemaps.usgs.gov/wnv_us_human.html (accessed November 23, 2012).

\section{AUTHOR BIOGRAPHIES}

MOHAMMAD F. OBEID is a $\mathrm{PhD}$ student and a research/teaching assistant in the department of Modeling, Simulation and Visualization Engineering at Old Dominion University. He received his MS degree in Modeling and Simulation from Old Dominion University and his BSc degree in Industrial Engineering from the German-Jordanian University. His research interests include modeling and simulation in healthcare, simulation optimization, biomedical engineering and surgical simulation. His email address is mobei001@odu.edu.

JOHN SHULL is a full time graduate student in the department of Modeling, Simulation and Visualization Engineering College at Old Dominion University. He received his BS in Business Administration from Old Dominion University. John is currently a free-lance consultant with his academic research interests in Agent Based Modeling and Agent Based Simulation. His email address is jshu1003@odu.edu. 